\title{
Human Papillomavirus Genomics: Past, Present and Future
}

\author{
Ariana Harari ${ }^{\mathrm{a}}$ Zigui Chen $^{\mathrm{b}} \cdot$ Robert D. Burk ${ }^{\mathrm{a}-\mathrm{d}}$ \\ Departments of a Microbiology and Immunology, bediatrics, ' Obstetrics, Gynecology and Women's Health, \\ and ${ }^{d}$ Epidemiology and Population Health, Albert Einstein College of Medicine, Bronx, N.Y., USA
}

\begin{abstract}
Human papillomaviruses (HPV) are a group of divergent DNA viruses, of which a select few evolutionarily related HPVs have emerged to be highly oncogenic and of significant medical importance. Essentially all cases of cervical cancer, as well as a subset of other anogenital and oral cancers are caused by this limited set of HPV types. At present, over 150 HPV types have been identified and may be classified into genera, species and types based upon comparison of the viral genome. Established nucleotide phylogenies sort the highly pathogenic HPV types to the genus Alphapapillomavirus (aPV). A species group includes viral types with 60-70\% genomic nucleotide similarity that share a most-recent common ancestor; for example the species group's alpha-9 (HPV16-related) and alpha-7 (HPV18-related), contain the majority of known oncogenic HPV types. Genomes from the same HPV type with 1-10\% nucleotide differences designate HPV variant lineages. The established nucleotide variations observed in extant HPV genomes have been fixed through evolutionary processes prior to human population expansion and global dissemination. To characterize viral types and variants associated with pathology for clinical applications (e.g. screening), molecular epidemiological studies have proven essential for identifying links between HPV natural history and carcinogenicity. This chapter presents a historical account of HPV genomics in the context of major discoveries and advances over the past 2 thousand years.

() 2014 S. Karger AG, Basel
\end{abstract}

Papillomaviruses (PVs) are ubiquitous, highly diverse DNA viruses that have been isolated from all 4 classes of Tetrapoda, including most mammals as well as birds, turtles and snakes [1-3]; their origin predates the existence of modern humans [4-7]. Observational accounts of warts from ancient Greece and Rome describe condylomatous lesions on the skin and genitals, and it was presumed that genital warts were associated with promiscuous sexual behavior. Warts in general were surmised to be transmissible [8]. Manifestations of animal PVs have historically been documented in myths and paintings, particularly of the 'jackalope'. This animal does not exist, but likely represents a case of mistaken identity as a result of a PV infection that produced 
cornified growths (i.e. horns) on jackrabbits. Historical records and myths provide evidence for the antiquity of PVs and the diseases they cause. In 1842, the Italian physician Dr. Rigoni-Stern was the first to hypothesize that cervical cancer might be linked with sexual behavior. He observed that cervical cancer frequency was disproportionally higher in prostitutes and married women than nuns and virgins, implying that the causative agent was likely sexually transmitted $[9,10]$. Rigoni-Stern's observation would be validated almost 150 years later; today, human PVs (HPVs) are known to be among the most commonly sexually transmitted infections, and infections by specific high-risk (HR) HPV types are known to be the etiological agents of cervical cancer [11-14].

In 1911, Francis Peyton Rous famously demonstrated that filtered tumor cell extract obtained from chicken carcinomas and transplanted to naïve chickens promoted sarcoma growth of a virulent nature, identifying the first oncogenic virus, i.e. Rous sarcoma virus. Almost 25 years later, Dr. Rous and Dr. Richard Shope identified the first PV, known as Shope PV or cottontail rabbit PV, from warty growths on cottontail rabbits. They went on to demonstrate that transmission of cottontail rabbit PV exhibited neoplastic potential in domestic rabbits $[15,16]$. Approximately 75 years later, Prof. zur Hausen was awarded a Nobel Prize for the discovery of HPVs causing cervical cancer $[17,18]$. The discovery that HPVs are major contributors to cancer and represent highly adaptive, carcinogenic viral pathogens causing essentially all of cervical cancer and approximately $20 \%$ of head and neck cancers has energized the research and medical communities [14] (fig. 1).

Cervical cancer ranks 3rd amongst cancers affecting women worldwide and 2nd in developing countries [14]. Women in developing countries account for $85 \%$ of the global incidence of cervical cancer. Incidence rates are nearly double in developing compared to developed countries, 17.8 and 9.0\%, respectively. This difference is thought to be largely due to the implementation of early diagnostic screening methods, which have reduced the risk of cervical cancer associated with persistent HPV infection. Yet, cervical cancer is still responsible for 275,000 deaths/year [14].

HPV infection by any of the 150 identified HPV types is not sufficient to cause cancer. All genital oncogenic types belong to the genus Alphapapillomavirus, which is currently comprised of 62 known HPV genotypes that infect the mucosal epithelium. Further classification beyond the genus level is required to distinguish the 1315 HR HPV types that are associated with oncogenic risk. It is well established that phylogenetic analyses cluster HPV taxa by host cell tropism (e.g. skin vs. genitalia), degree of oncogenic potential and morphology of clinical lesions [1, 19]. Progression to cancer is rare. The malignant potential of specific HPVs likely results from niche adaptation (i.e. evolution of an organism/virus to a specific biological/anatomical ecosystem on the body) of PVs, as cancer is undesirable for both the virus and its host. The majority of HPV infections are cleared within 6-10 months. Persistent infections with HR HPV sare a critical risk factor for the development of HPV-associ- 


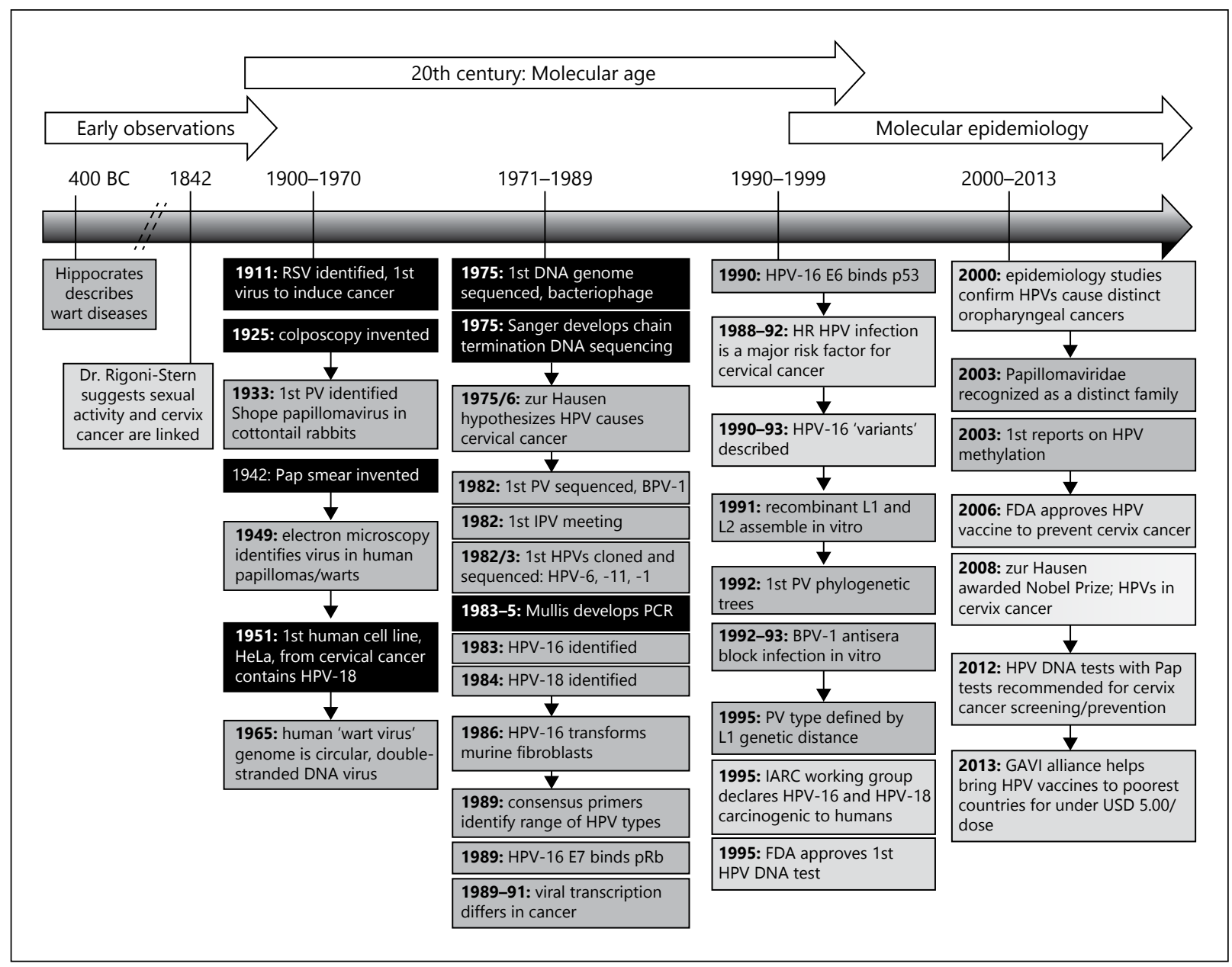

Fig. 1. History of HPV, cervix cancer and technological advances. The timeline displays the landmarks in PV biology and clinical discovery. The color of the boxes serves to distinguish events and/or discoveries relating to basic PV scientific advancements in red, biological discoveries/technology innovations in blue, or epidemiology/public health in green. RSV = Rous sarcoma virus; BPV = bovine PV; IPV = International Papillomavirus meeting; $\mathrm{pRb}=$ retinoblastoma protein; IARC = International Agency for Research on Cancer; FDA = Food and Drug Administration; GAVI = Global Alliance for Vaccines and Immunization.

ated precancer and cancer [20-22]. Delineating the differences intrinsic to these HR HPV genotypes, compared to the majority of HPV types that lack oncogenic potential, will help to elucidate the genetic basis of such carcinogenic properties, thus contributing to a better understanding of the biological mechanisms exploited by the virus to facilitate cancer development. Epidemiology studies provide a platform for obtaining viral isolates used to investigate the dynamic relationship of HPV genotype differences and clinical disease. The recent explosion in DNA sequencing tech- 
nologies will continue to revolutionize methods of HPV detection [23] contributing to a better understanding of HPV biology and the development of new therapies against HPV-associated cancers.

\section{Human Papillomavirus Genome Characteristics}

$\mathrm{HPV}$ is a circular, nonenveloped, double-stranded DNA virus approximately $8 \mathrm{~kb}$ in size that infects basal keratinocytes. Upon infection, the virus exists as an autonomous episome in the host cell nucleus. The viral life cycle is mediated by a series of virushost interactions, which govern viral transcription, virion production and eventual clearance in the majority of infections [24, 25] (fig. 2).

The structure and function of the HPV genome are conserved throughout the Papillomaviridae and are broadly divided into 3 general components. (1) The early gene region, denoted by 'E', consists of 6 open reading frames (ORFs): E6, E7, E1, E2, E4, and E5. The early genes E1, E2, E6 and E7 are generated as a polycistronic transcript. Several additional early ORFs E3, E5 and E8 have also been identified, but their expression is not uniformly observed throughout the Papillomaviridae. Viral transcripts can undergo extensive alternative splicing, contributing to the intricate balance between viral and host-regulated transcription [for a review, see 26]. The early genes code for nonstructural proteins that function in viral replication, adaptation of the cellular milieu for viral activities, trans-activation of viral transcription and cellular transformation and proliferation. (2) The late gene region, denoted by 'L', consists of the L2 and L1 ORFs. L1 and L2 encode the structural proteins, the major and minor capsid proteins, respectively. The L2 ORF encodes for group-specific epitopes whereas the L1 ORF contains type-specific protein domains. (3) The upstream regulatory region (URR) is the noncoding region between the end of the L1 ORF and the E6 start codon, comprising approximately $10 \%$ of the genome. The URR contains DNA recognition sites for both viral and host transcription factors and regulates early gene transcription, viral amplification and cellular tropism. The URR contains a keratinocyte-specific enhancer region proximal to the early gene promoter (p97), which highlights the significance of host cell tropism to viral gene expression and life cycle. A smaller noncoding region located between the E5 stop and L2 start codons harbors a highly conserved early polyadenylation signal required for gene expression from the early promoter, including alternatively spliced early transcripts and their gene products [26]. Both the early gene region and the URR display variability, useful in assessing genetic heterogeneity [27]. Increased understanding of the inherent genomic differences between HPV species that contribute to viral function is predicated on biochemical techniques such as DNA sequencing and polymerase chain reaction (PCR) which have contributed greatly to understanding the molecular pathogenesis of HPV. Great strides have been made since the inception of clinical HPV molecular biology in the 1970s [7, 28-30]. 


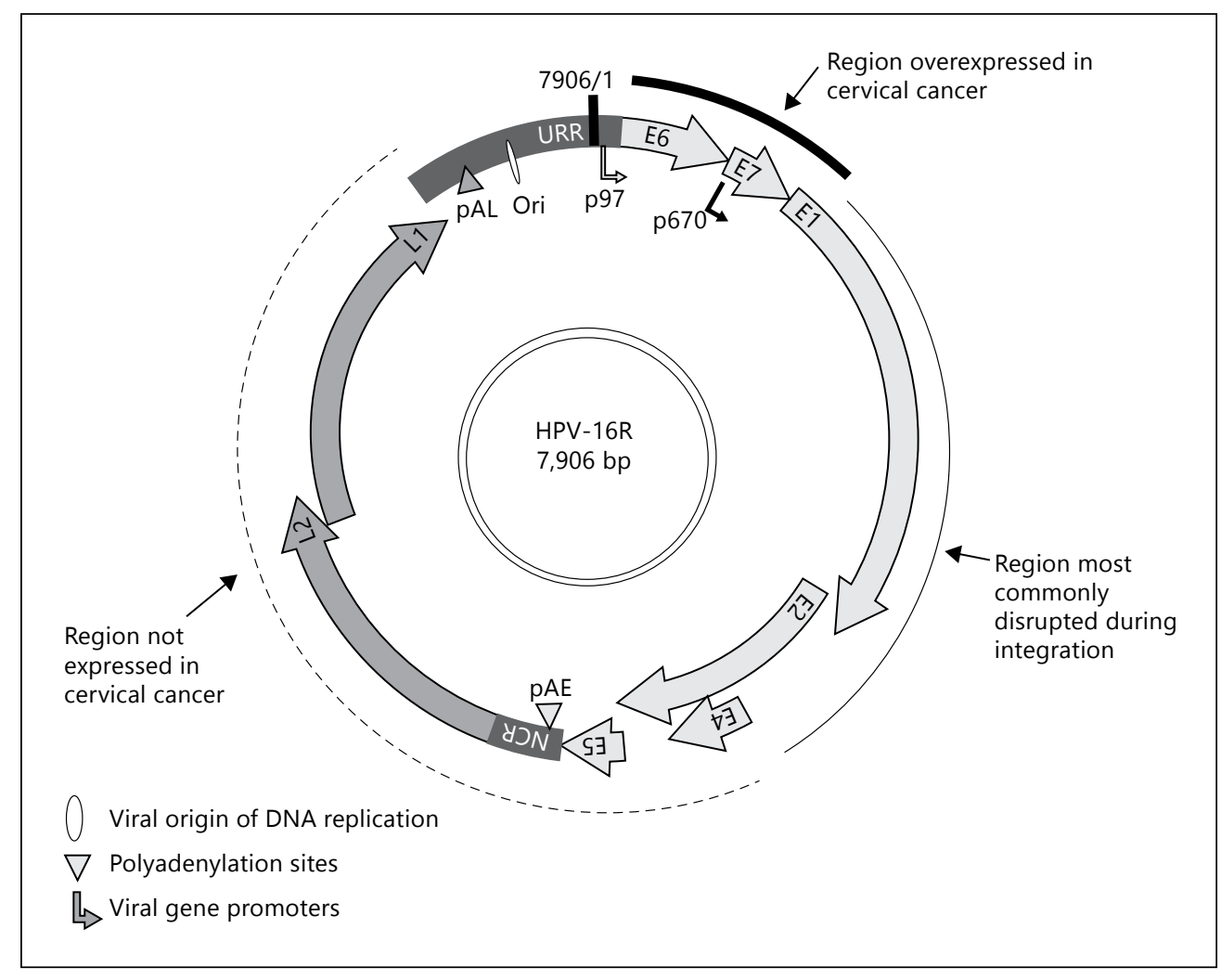

Fig. 2. Schematic of the HPV-16R genome. The HPV-16 genome displayed shows the general organization of the HPV open reading frames (ORFs) and regulatory regions. Early (E) genes and related transcriptional regulatory motifs are shown in green; late $(\mathrm{L})$ genes and related transcriptional regulatory motifs are shown in blue; the 2 noncoding regions are shown in gray; the smaller region between $\mathrm{E} 5$ and $\mathrm{L} 2$ is termed the noncoding region (NCR), and the larger region between L1 and E6 is known as the upstream regulatory region (URR). The URR contains regulatory elements including the viral DNA origin of replication (Ori) depicted by a yellow oval and the early gene promoter p97 depicted by a green arrow. The blue arrow in the E7 ORF depicts late gene expression regulated by the late promoter $\mathrm{p} 670$. Polyadenylation sites termed early $(\mathrm{pAE})$ or late $(\mathrm{pAL})$ are shown as green or blue triangles, respectively. Arrowheads at the tip of each ORF depict if they overlap. The thick solid line represents genomic regions commonly overexpressed in cancer, the thin black line represents genomic regions frequently disrupted by integration, and the dashed line represents the genomic region rarely expressed in cancer.

The viral proteins E1 and E2 function in viral genome replication and are dependent on the host DNA polymerase and replication machinery. E1 functions as an ATP-dependent helicase capable of melting double-stranded DNA for strand separation, prior to DNA polymerization. The E2 ORF encodes for a viral-DNA binding transcription factor. E1 and E2 proteins form heterodimers at the viral origin of replication to initiate bidirectional genome synthesis. Recently, E1 and E2 have been shown to function in the early induction of the DNA damage response pathway con- 
tributing to a permissive environment for viral genome amplification [31]. E1 can induce beaks in the host double-stranded DNA that activates the ataxia-telangiectasia DNA damage response pathway, signaling cell cycle arrest [31]. The URR contains 4 highly conserved E2 binding sites that differentially regulate viral replication and early gene transcription $[32,33]$. E2-dependent downregulation of early promoter activity maintains low-copy numbers of viral genomes prior to differentiation dependent activation of the late promoter and genome amplification. In high-grade neoplastic lesions/cancer the E2 ORF can be disrupted by viral integration into the host genome. Integration results in the loss of E2-dependent early promoter regulation, a ramification of which can be overexpression of E6 and E7 [24, 25, 34]. The viral proteins E6 and E7 function as oncogenes in the HR $\alpha$-HPVs. HR HPV E6 and E7 proteins disrupt cell cycle regulation in upper epithelial cells (stratum spinosum), which normally exit the cell cycle to terminally differentiate. Virally mediated inactivation of key cell cycle regulators, the known tumor suppressors, p53 and pRb (by E6 and E7, respectively), are distinct among certain $\alpha-\mathrm{PV}$ types, although not specific to those causing cancer [35]. The E5 ORF encodes a transmembrane protein that probably contributes to cell signaling [36]. The E5 ORF as defined by the presence of a true start and stop codon is found in select members of the human $\alpha$-PVs: the HR HPV species, the $\alpha-10$ species and other PVs including bovine PV 2 implicated in urinary cancer (cattle). At the nucleotide level, E5 is not highly conserved. The E5 protein is associated with late gene viral life cycle events and interacts with epidermal growth factor and platelet-derived growth factor to influence cellular proliferation [37]. The integrity of the E5 ORF provides another example of the differences revealed by studying HPV genotypes and phylogeny [38]. The late genes L1 and L2 function in virion maturation and orchestrate virion self-assembly that packages the genome for release in the upper epithelia. During late stages of precancer/cancer, L1 and L2 proteins are not expressed. This initially made early studies on HPV identification difficult and required the use of extracted DNA from warts to be obtained for analysis. Virus-like particles made from HR HPV L1 readily self-assemble and induce a neutralizing antibody response. This is the basis for the two prophylactic vaccines currently available.

\section{Viral Identification and Classification: Phylogeny and Taxonomy}

HPV genome heterogeneity was apparent during the initial studies examining viral isolates obtained from dysplastic lesions (cutaneous or genital). By the late 1970s, an unknown viral agent was known to be the causative agent implicated in the development of multiple wart types, condylomata acuminata (genital warts) and possibly cervical cancer. Attempts to identify the viral agent from lesions were hampered by the fact that DNA probes often did not cross-react with DNA extracts obtained from different wart types, implicating the presence of diverse viruses [39]. Furthermore, early attempts to study the infectious nature of the virus obtained from warts was restricted 
by the highly specific host epithelial-cell specificity required for a productive HPV life cycle $[30,40]$. Understanding the delicate balance of HPV-host interactions proved fundamental to addressing the clinical significance of HPV-related disease.

HPVs are extremely ancient viruses, and related PVs have been identified in most nonhuman primates, including humans' most recent common ancestors [41-43]. Sequence and phylogenetic analysis of nonhuman primate PVs isolated from the cervicovaginal area revealed genomic similarity to the $\alpha$-HPVs. Several nonhuman primate PVs identified from rhesus macaques (Macaca mulatta), cynomolgus macaques ( $\mathrm{Ma}$ caca fascicularis), and olive baboons (Papio hamadryas anubis) comprise the $\alpha-12$ species. These phylogenetically related $\alpha-P V$ types can induce epithelial dysplasia of varying degrees, resembling the high-grade cervical intraepithelial neoplasia (CIN) associated with persistent HR mucosal-HPV infections, specifically within the a-9 species, which includes HPV-16 [5, 6, 44]. Experimental transmission of $M$. fascicularis PV-3 from a naturally infected female to naïve female macaques was associated with the development of CIN [45]. These nonhuman primate PVs are more similar to the $\alpha-9$ species, than more distantly related HPV types, such as the $\alpha-10$ species, suggesting that the mechanisms governing cellular transformation result from a common ancestral trait that predates human/primate divergence.

Diversification of HPVs occurred prior to the emergence of Homo sapiens approximately 200,000-150,000 years ago [46]. The extensive diversification and demographic range of HPVs reflect human dispersal and population expansion [4], and may intuitively suggest that HPVs have had to undergo a high rate of mutations to adapt to such diverse hosts; however, this is not the case. Rates of nucleotide mutations are remarkably low in the virus, observed at a rate of approximately $10^{-8}-10^{-7}$ nucleotides/year [47].

HPV characterization in population-based studies affords the unique opportunity to study hominid evolution through a viral lens to better understand virus-host interactions as they pertain to immune system surveillance and cancer progression $[5,6$, 38]. The unique coupling of the PV-host-dependent life cycle has selected for specialized viral adaptation to specific host niches, coincident with the ability of the virus to evade a host immune response. These features have enabled PVs to capitalize on their hosts, and exploit global diversity to thrive throughout evolutionary time. Genital HPV infections are commonly dependent on sexual transmission. This exemplifies one way the virus invests in its existence; they have hijacked the most fundamental aspect of host species success, reproductive fitness. After infection, viral gene expression is tightly regulated such that it is dependent on host epithelial differentiation for near exclusive activation of either the early or late gene promoters driving gene expression. Such adaptation tactics suggests a commensal virus-host relationship, yet HPV-dependent malignancies defy this viewpoint. Molecular epidemiological studies assessing the phylogenetic association of HPVs based on oncogenic risk support specific biological and pathological traits distinct to HPV genera, species and types [38]. HPV-16 is unique in its ability to establish persistence that is highly associated with 
neoplastic progression, both of the cervix and in head and neck carcinogenesis. The a-HPVs exhibit agreement between (clinical) natural history studies and HPV phylogeny and taxonomy, providing evidence to support that carcinogenicity is an evolved trait most probably related to niche adaptation [38, 48-50].

\section{Human Papillomavirus Classification}

Methods to culture HPV in vitro or produce infectious virus through xenotropic models are not efficient, and do not provide a robust method for identification and characterization of HPV types [51]. Furthermore, a lack of a robust, consistent antibody response in infected individuals limits the use of antibody titer and serology for HPV taxonomy [51]. Historically, HPV has been identified from biopsies of warts or lesions and classified by comparison to known types by restriction endonuclease cleavage patterns and/or DNADNA or DNA-RNA blot hybridization. Such methods had innate flaws for characterizing PVs; specifically there was no quantifiable means of comparison amongst HPV types from different lesions, the virus titer in lesions was not available, and cross-hybridization was difficult to explain except for association by anatomical site [30, 40]. The appreciation for a genomic, DNA-sequence-based classification system was agreed upon by the PV research community by the late 1980s. This system has relied on the rapid advances in DNA technologies from PCR to the Next-Gen sequencing era upon us.

Today, PCR-based amplification of DNA obtained from clinical samples is common. As the realization that phylogeny and genotyping methods validated one another, the notion that only a limited set of HPV types were associated with cancer spurred the need for highly specific assays that could discriminate HPV types. Consensus PCR primers targeting highly conserved regions within the L1 ORF, such as the MY09/11 PCR assay or the GP5+/GP6+ PCR assays, are typically used for HPV identification [52]. A review of HPV detection methods has recently been published [53]. In addition, sequencing of the PCR amplicons for alignment to known HPV type(s) facilitates classification of genotype by nucleotide identity and identification of novel types.

PVs belong to the family Papillomaviridae and were given this status by recognition of a genome-based, DNA sequence system for classification [54]. DNA sequencing provides a quantifiable means to catalog nucleotide heterogeneity, affording the classification and taxonomy of HPV to genera, species, types and variant lineages [27, 55]. Classification of HPV genera and species based on DNA sequence was recognized by the PV Working Group at the 14th International Papillomavirus conference in Quebec in 1995, later adapted by the International Committee on the Taxonomy of Viruses $[1,19,51,56]$. Variant lineage classification is a more recent development within the PV community that will become increasingly more relevant as high-resolution techniques, such as nextgeneration sequencing, generate a plethora of PV sequencing data that need to be coherently analyzed, named and correlated with phenotype and geographic locations [55]. 


\section{Human Papillomavirus Genome 'Typing'}

The L1 gene is highly conserved and provides the basis for HPV genotyping. An HPV 'type' is designated when the nucleotide sequence of the L1 ORF from the cloned viral genome is more than $10 \%$ dissimilar to all known types [1].

Nucleotide differences across HPV genotypes are correlated with viral lineages based on evolution without significant, if any, recombination, and these correlated changes are the result of lineage fixation [57]. Whole genome sequencing established L1 sequence identity as representative of complete genome variation due to its high conservation $[1,50]$. Nucleotide sequences are used to build phylogenetic trees used for HPV taxonomy. Phylogenetic analysis suggests the underlying relationship between the biological observations identified within this heterogeneous group of viruses: including host cell tropism (mucosal or cutaneous), carcinogenic risk and associated pathology [38]. Currently, over $150 \mathrm{HPV}$ types have been formally identified and predominantly cluster to 3 main genera. (1) Alphapapillomavirus are primarily isolated from the genital, mucosal epithelium and are the overwhelming cause of anogenital cancer; (2) Betapapillomavirus are primarily isolated from skin lesions. The $\beta$-PVs include HPV types frequently associated with the rare genetic disorder epidermodysplasia verruciformis (EV), which predisposes individuals to develop HPV-associated cutaneous, scaly wart-like squamous cell carcinomas. Many $\beta$-HPV types were originally identified in isolates obtained from EV patients, previously termed HPV EV types and include HPV- 5 and HPV-8, both members of the $\beta-1$ species, identified in approximately $90 \%$ of EV-related cutaneous squamous cell carcinomas. Less prevalent EV types extend to the $\beta-2$ species (HPV-38) and $\beta-3$ species (HPV-49). These types are also found associated with malignancy in immunocompromised hosts, and are less prevalent in the general population [58]. (3) Gammapapillomavirus are primarily isolated from cutaneous epithelia, some from cutaneous lesions histologically defined by the presence of homogeneous intracytoplasmic inclusion bodies $[1,59]$. Both Gammapapillomaviruses and Betapapillomaviruses have been identified in oral samples suggesting they have an expanded tropism including the oral cavity [60] (fig. 3). Thus, the tropism of these later genera has to be reconsidered in light of the new information. This also demonstrates that not testing a specific anatomical site (e.g. the oral cavity) for HPV (using methods to detect the gamut of types) does not mean the virus is not there.

\section{$\alpha$-HPV Phylogenetic Association with Cancer Risk}

Phylogenetic analysis based on the sequence of the HPV L1 ORF has been the standard for genomic analysis and type classification $[1,19,54]$. Observations of phylogenetic incongruence within the $\alpha-\mathrm{PVs}$ are shown by comparison of trees built utilizing either the early or late regions of the genome. This results in differences regarding the monophyletic origin of the oncogenic $\alpha$-HPV types. Trees generated from the early genes or the complete genome cluster $\alpha-\mathrm{PVs}$ by associated oncogenic risk as a mono- 


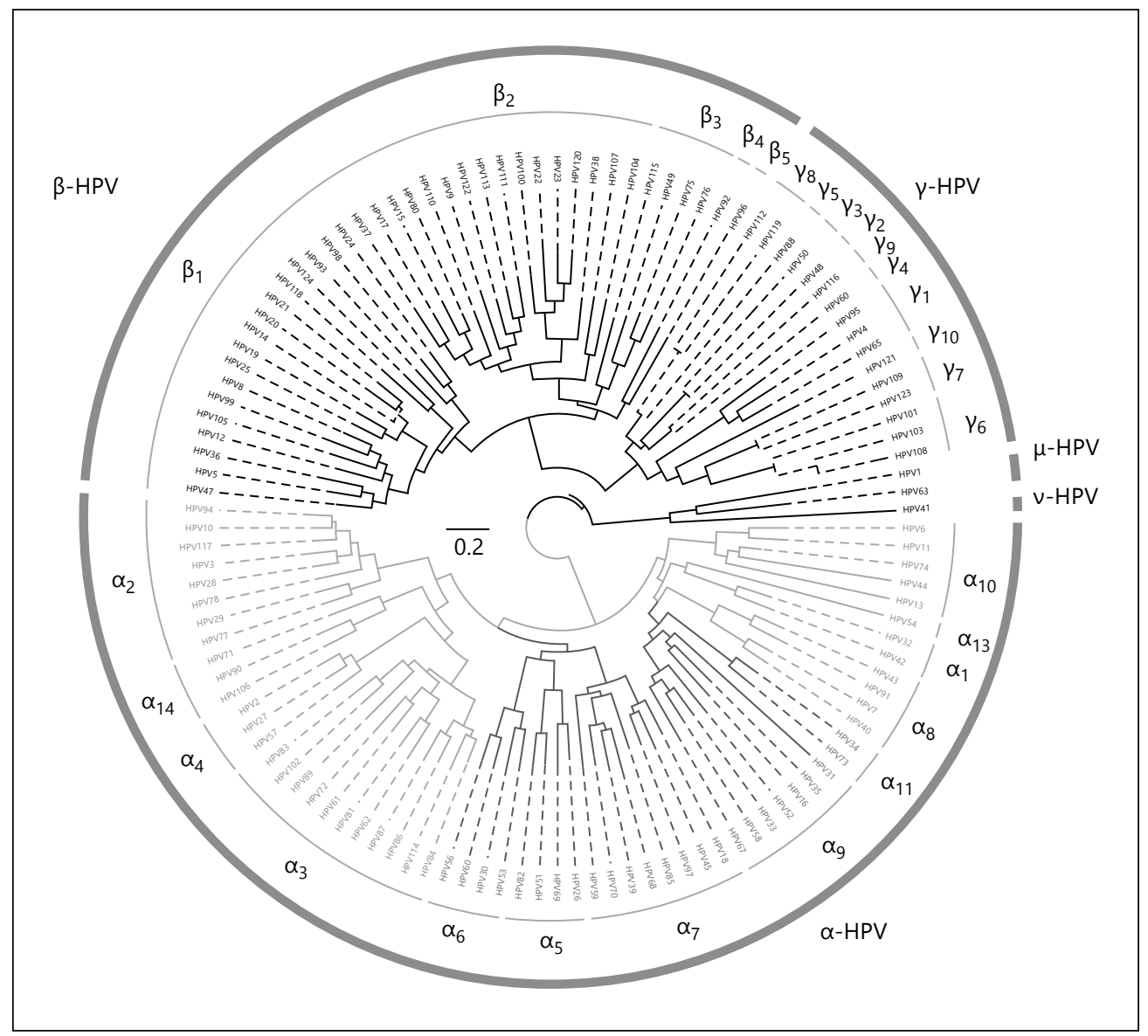

Fig. 3. HPV phylogenetic tree clustering the majority of HPVs into 3 genera, Alphapapillomavirus, Betapapillomavirus and Gammapapillomavirus. A maximum likelihood tree was constructed using RAxML version 7.2.8.27 [80] and an alignment of the nucleotide sequences of the L1 ORF of 120 published HPV types. HPV species groups were generally classified according to the classification system for PVs by Bernard et al. [19]. All HR mucosal HPV types cluster within the genus Alphapapillomavirus, highlighted in red. The clades in blue represent mucosal HPV types with low risk or no risk of cervical cancer. The scale bar represents a nucleotide change of 0.2 per site.

phyletic clade. Phylogeny based on late gene regions (L1 and L2) does not support a monophyletic origin for the 5 oncogenic $\alpha$-HPV species ( $\alpha 5, \alpha 6, \alpha 7, \alpha 9, \alpha 11)$. This phylogenetic incongruence is suggestive of genomic distinctions within the $\alpha-\mathrm{PVs}$ that are exemplified by examining differences inherent to the species $\alpha 9$ (HPV-16-related) and $\alpha 7$ (HPV-18-related) that differ in biology and pathological outcome. HPV-16 and HPV-18 are highly prevalent, oncogenic types implicated in $70 \%$ of cervix cancer, and as members of different species groups they exhibit different biological niches and cellular tropisms, and manifest as precancerous and/or cancerous lesions differently. HPV-16 targets the cutaneous squamous epithelia abundant in the 
ectocervix and predominantly causes cervical squamous cell carcinoma that evolves through differing grades of squamous intraepithelial neoplasia observed by histological/cytological screens [61]. HPV-16 also infects the squamous epithelia of the oropharynx and is identified in oropharynx cancer (targeting the regions of the throat including the base of the tongue, the soft palate and the tonsils). Additionally, HPV-16 is implicated as causal agent for cancers of the vulva, vagina, penis and anus [18]. Conversely, HPV-18 disproportionately targets glandular, mucin-secreting columnar epithelial cells found primarily in the endocervix, and is overrepresented in the development of cervical adenocarcinoma $[62,63]$.

Comparison of phylogenetic trees generated from either early or late gene sequences results in different phylogenies for distinguishing higher-level taxa [48]; the clades defining species groups remain intact. Yet, the nodes defining a clades' most recent common ancestor appear differently depending on the genes used for the phylogenetic tree construction, reflecting a degree of phylogenetic incongruence. This hints at the occurrence of early selection events likely driven by ecological niche adaptation such that early and late genes are regulated through distinct promoters that are strictly governed by the availability of host proteins differentially expressed within the stratified epithelia $[25,48]$. Incongruence may also result from disproportionate selective pressures on the two genomic regions. Incongruence does not likely result from an early viral recombination event, as similar genome characteristics are maintained across diverse hosts throughout evolutionary time, such as humans and nonhuman primates, supporting the clonal nature of viral expansion as opposed to recombination $[38,48]$.

\section{Variant Lineages: Model for Recent Human Papillomavirus Speciation}

Classification below the species level is not formally recognized by the International Committee on the Taxonomy of Viruses $[19,55]$. Evidence from large epidemiological studies identifying HPV genomic heterogeneity and associated pathologies supports the need for distinction of taxa below the HPV type level and will likely be updated soon [55]. The term HPV subtype has become obsolete. It previously referred to isolates that exhibited $2-10 \%$ differences in nucleotide identity compared to its closest known type and/or differences in restriction enzyme cleavage patterns [1]. As better systems for identification and classification of HPVs emerged, subtypes are now considered variant lineages.

\section{Variant Lineages and Sublineages: Updates on Current Nomenclature Guidelines}

$\mathrm{HPV}$ variant lineages represent viral isolates that exhibit genomic nucleotide differences of $1-10 \%$, as compared to their prototype, or reference genome $[5,6,27,50,55$, 64]. Viral variants share a most recent common ancestor that is unique to the specific HPV type. HPV variants are common and may differ in the risk for development of 
cervical precancer (CIN2/3) or cancer. Initial studies examining HPV-16 and HPV-18 intratypic variation aimed to discern the contribution of variant lineages to geographic differences in virus distribution $[5,6,65]$. Variants of both HPV-16 and HPV-18 were initially classified by sequencing the URR, as this noncoding region exhibits greater nucleotide variability than protein-coding regions and is a valuable tool for identifying stable nucleotide polymorphisms, the basis for HPV classification. However, unlike type identification that is sufficiently designated by sequencing the L1 ORF, complete genome sequencing is required to identify HPV variants since the distribution of differences is not evenly spaced across the genome. In addition, a variant nomenclature system based on the complete genome permits the identification and quantification of nucleotide polymorphisms using different regions of the genome [55]. Evidence supports that certain genomic regions exhibit greater heterogeneity than others.

HPV replication depends on host DNA replication machinery; proofreading capabilities by host DNA polymerases maintain a low rate of mutation within the viral genome at approximately $10^{-8}-10^{-7}$ nucleotide substitutions/year [4]. Intratypic HPV genetic variation results from random nucleotide polymorphisms or insertions/deletions (indels) acquired through genetic drift or natural selection that become fixed over time. Stable acquisition over time, of these nucleotide changes, eventually leads to PV speciation through a process termed lineage fixation [57] and is further supported by a lack of evidence for viral recombination events [49]. Furthermore, the stable acquisition of polymorphisms among isolates of the same HPV type (sharing a most recent common ancestor) eventually leads to type speciation, characterized by genomic nucleotide identity that is less than $90 \%$ and occurs over millions of years [46]. To this end, evidence of prehistoric human population bottlenecks is reflected through inter- and intratypic PV genomic diversity. Distinction below the species level (PV type) is common within the PV research community, and is useful for physicians, researchers and epidemiologists investigating HPV variants for association with geographical host population and viral genetic changes that confer variable phenotypic outcomes, including varying pathological manifestations such as cancer. At present, guidelines for variant HPV lineage classification are beginning to be formally established [55]. A formal classification and nomenclature system to describe intratypic HPV variants, at the lineage or sublineage level, will undoubtedly become increasingly useful as the future of HPV genomics expands to include data obtained from next-generation sequencing and metagenomic studies, and will facilitate a better system for cataloguing genotype-phenotype changes.

\section{Variant Lineages and Sublineages: Identification and Clinical Relevance}

Variant lineage classification is based on isolates of a known type that have had their complete genome sequenced and reveal genetic heterogeneity of at least $1 \%$ and less than $10 \%$, based on multiple sequence alignments to the prototype (first characterized genome of a given type). Parameters for variant classification have recently been established by phylogenetic analysis on isolates from the $\alpha_{9}$-species (including 
Table 1. HPV taxa definition and nomenclature overview

\begin{tabular}{llll}
\hline Taxa & Defining genomic characteristics & Region & Example (HPV-16 reference genome) \\
\hline Family & genome size approx. $8 \mathrm{~kb}$, distinct genome organization & L1 & Papillomaviridae \\
Genus & $>40 \%$ difference between genera & L1 & Alphapapillomavirus \\
Species & $30-40 \%$ difference to other types & L1 & Alphapapillomavirus 9 \\
Type & $>10 \%$ difference to known types & L1 & HPV-16 \\
Lineage & $1-10 \%$ difference to same type & CG & variant lineage A \\
Sublineage & $0.5-1 \%$ difference to same type & CG & variant sublineage A1 \\
\hline
\end{tabular}

CG refers to the complete genome sequence used for identification and nomenclature. The table lists the current taxonomic distinctions used for classification of PVs illustrated by the HPV-16 reference genome. The defining genomic characteristics refer to the aligned nucleotide sequence identity differences based on either the sequence of the L1 ORF or complete genome; these values are used to distinguish HPV taxa. The proper taxonomic orthography is shown for the HPV-16R genome. The inclusion of lineage and sublineage designation is not currently recognized by the International Committee on the Taxonomy of Viruses, but represents important taxa distinction recognized by the PV community.

HPV-16, HPV-31, HPV-33, HPV-35, HPV-52, HPV-58 and HPV-67), types from the $\alpha-7$ species and two types from the $\alpha-10$ species, HPV6 and HPV11 [27, 55, 64]. A divergence rate of $1 \%$ conservatively designates variant lineages. Similarly, pairwise nucleotide identity differences in the range of $0.5-1 \%$ define a type sublineage. Nomenclature for lineage and sublineage is based on an alphanumeric system wherein the prototype 'reference' genome is always designated with an 'A'. If sublineages for the given type are present, the prototype reference sequence is designated as 'A1' (table 1).

Variant lineages from many clinically relevant $\alpha-H P V s$ are known. Variants have been characterized for the majority of the HR mucosal HPV types: HPV-16 [5, 65, 66], HPV-18 [6, 46, 64], HPV types 31, 33, 35, 52, 58, and 67 [27]; other $\alpha-H P V$ variants that have been identified include members of the $\alpha-4$ species, i.e. HPV-2, HPV-27 and HPV-57 [67], and two types from the low-risk $\alpha-10$ species, specifically HPV-6 and HPV-11 [55], associated with genital warts have been extensively examined.

Phylogenetic analysis of HPV-16 variation revealed variants reflective of human dispersal out of Africa and divergence into the 3 major human races, Africans, Caucasians and Asians. Initial phylogenetic studies identified 4 major HPV-16 variants representative of host geographic origin. The major HPV-16 variants were broadly termed 'European' now lineage 'A' or 'non-European' now lineages 'B, C and D'. The non-European lineages are more heterogeneous and included 2 African HPV-16 lineages, African-1 and African-2 (B and C, respectively), and Asian-American variants (lineage D) $[5,57,62,68]$. These variants display phylogenetic congruence with host ethnicity and geographic origin [5]. However, with the recent update to nomenclature the designation of variant lineage simply (for the case of the HPV-16 example) as nonEuropean or European is misleading and overlooks details within the viral genomes (fig. 4). Previously termed HPV-16 variants have thus been renamed according to the 


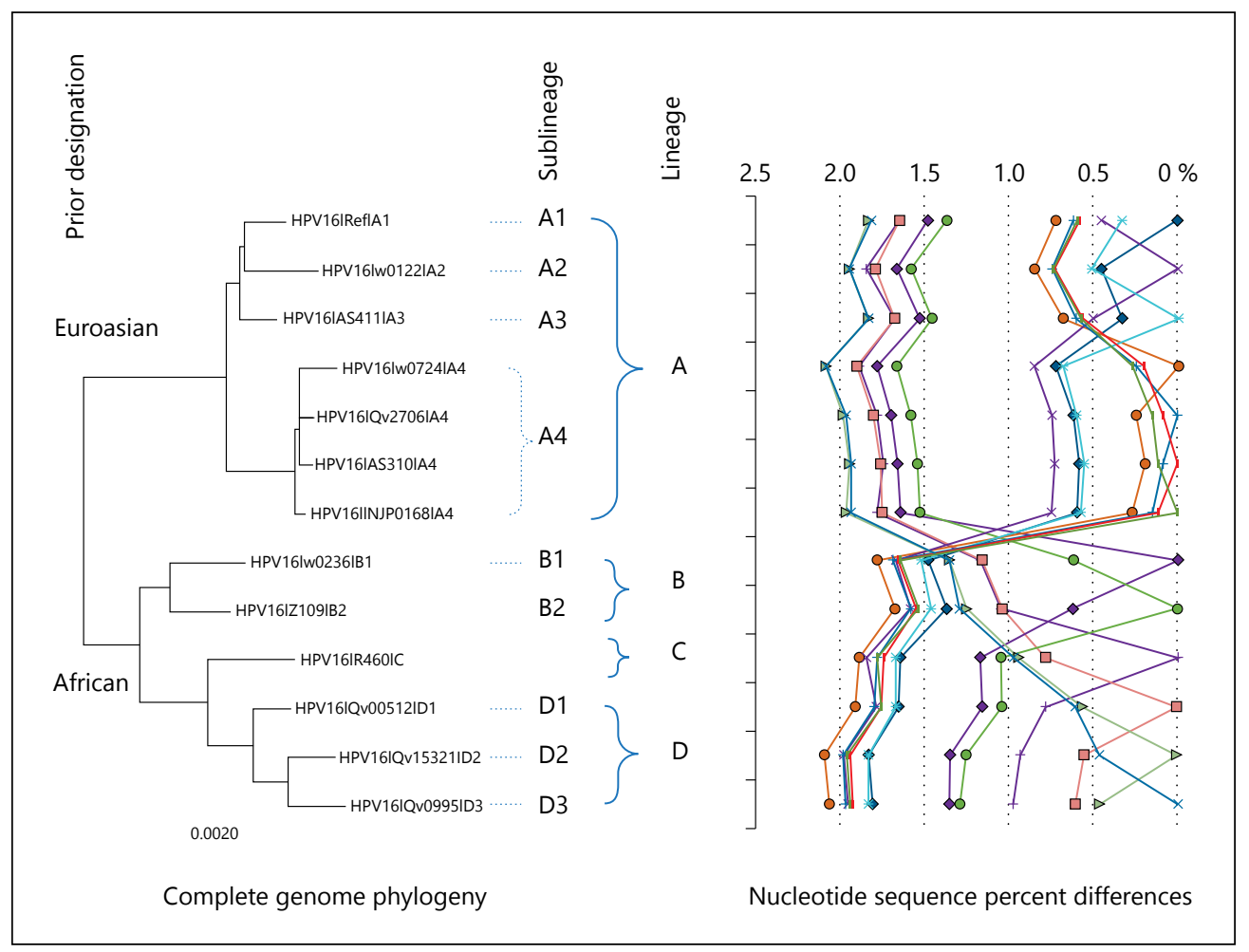

Fig. 4. HPV-16 variant tree topology and pairwise comparisons of individual complete genomes. A maximum likelihood tree was inferred from a global alignment of 13 complete nucleotide sequence genomes of HPV-16 using RAxML version 7.2.8 [80]. Distinct variant lineages (i.e. termed $A / B / C / D$ ) and sublineages (e.g. termed $A 1 / A 2 / A 3 / A 4)$ were classified according to the topology and nucleotide sequence differences from $>1 \%$ to $<10 \%$, and $>0.5 \%$ to $<1 \%$, respectively $[19,27]$. The percent nucleotide sequence differences were calculated for each isolate compared to all other isolates based on the nucleotide sequences (complete genome) and are shown in the panel to the right of the phylogeny. Values for comparison from an isolate are connected by lines, and the comparison to self is indicated by the $0 \%$ difference point. Symbols and colored lines are used to distinguish each isolate. The scale bar at the bottom of the tree represents a nucleotide change of 0.002 per site. Multiple isolates for the HPV-16 A4 sublineage are shown to highlight the intrasublineage relationship; note the clustering in the right hand graph that depicts nucleotide sequence differences.

updated variant nomenclature guidelines, whereby European variants are classified as the 'A' lineage, further resolved to 4 sublineages (A1, A2, A3, A4). The HPV-16 nonEuropean variants are now recognized as 3 distinct lineages with the appropriate sublineage designation: HPV16-B (previously Af-1) contains sublineages B1 and B2; HPV-16C (previously Af-2); HPV-16D (previously NA1, AA1, AA2) contains sublineages D1, D2 and D3. This update helps to resolve and term the viral distinctions below the type level and is important since many of the nucleotide changes are correlated within taxa (fig. 4). 


\section{The Future of Human Papillomavirus Genomics}

Biomedical Technology Advancements and Biomarker Prediction

Global epidemiological studies now permit access to vast numbers of clinical HPV samples to better understand the natural history of HPV infections [50] and the biological and clinical ramifications $[50,69]$. The risk associated with a persistent HR infection, such as HPV-16, is significantly associated with neoplastic progression. However, determinants of viral persistence and clearance are not well understood. Recent evidence suggests differences in the duration of persistence for different HPV types and/or lineages associated with clinical outcomes $[50,70,71]$. Understanding of HPV genomics and classification will help further characterize host/viral components involved in viral persistence and/or clearance $[72,73]$. This may contribute to the design of new treatments and therapeutics targeting those most at risk for cancer. On a broader scale, it may also help delineate which infections require treatment versus those that naturally regress, having implications on the financial burden associated with cancer prevention and treatment globally.

Rapid technology advances during the past decade have, and will continue to, advance our understanding of PV genomic heterogeneity that contributes to pathological clinical outcomes. Next-generation sequencing provides increased resolution for the detection and classification of viral DNA, at the single molecule level, and will enhance methods for identification of new HPV taxa that may have previously fallen under the limit of detection by current methods [74]. This will facilitate a more complete view of Papillomaviridae diversity and should contribute to an improved understanding of the mechanisms of HPV-associated malignancy $[75,76]$. Increased use of novel genotyping methods will improve the efficiency of identifying HPV DNA from an array of samples obtained by large cohort studies. The explosion in data generated by nextgeneration sequencing techniques reinforces the need for current, widely accepted methods to characterize the results. This is highlighted by recent reports describing an extensive array of novel Betapapillomavirus and Gammapapillomavirus types, which are currently difficult to classify given the extensive diversity of these types and the lack of a simple method for their characterization in a standard clinical assay.

New Codes in the HPV Genome

The appreciation of an additional 'epigenetic code' (i.e. CpG sites that can be methylated) in the HPV genome has energized new and future studies on understanding the significance of information. HPV epigenetics is a burgeoning field [for a review, see 77]. Accounts of CpG methylation of viral DNA, predominately from HPV-16 and HPV-18 were initiated at the turn of the 21 st century and identified regions of viral CpG methylation by methylation-specific restriction endonuclease maps. Different cleavage patterns from viral isolates obtained from women with different stages of HPV-associated CIN have been observed. The onset of technological advances within the past 2 decades has generated methods for more accurately identifying and quantitating DNA methylation. CpG sites are highly conserved amongst HPV species. CpG sites appear 
throughout the genome and exhibit varied methylation states and likely play a physiological role in the viral life cycle. Large epidemiological studies that aim to elucidate the oncogenic properties of HPV have demonstrated that methylation at specific CpG sites within the viral genome is predictive of clinical outcome, such as precancer (CIN2/3) or cancer [77-79]. Specifically, CpG sites found within the HPV-16 L1 ORF are highly predictive of CIN2/3 [78]. Interestingly, the $\alpha-7$ species contain higher numbers of CpG sites relative to the $\alpha_{9}$-species. The development of high-resolution methods for HPV identification and classification should lead to the discovery of additional coded information in the HPV genome beyond nucleotide, amino acid, CpG and DNAbinding protein regions.

\section{References}

$>1$ De Villiers EM, Fauquet C, Broker TR, Bernard HU, zur Hausen $\mathrm{H}$ : Classification of papillomaviruses. Virology 2004;324:17-27.

- 2 Herbst LH, Lenz J, Van Doorslaer K, et al: Genomic characterization of two novel reptilian papillomaviruses, Chelonia mydas papillomavirus 1 and Caretta caretta papillomavirus 1. Virology 2009;383:131-135.

-3 Lange CE, Favrot C, Ackermann M, Gull J, Vetsch E, Tobler K: Novel snake papillomavirus does not cluster with other non-mammalian papillomaviruses. Virol J 2011;8:436.

4 Bernard HU: Coevolution of papillomaviruses with human populations. Trends Microbiol 1994;2:140143.

$>5$ Ho L, Chan SY, Burk RD, et al: The genetic drift of human papillomavirus type 16 is a means of reconstructing prehistoric viral spread and the movement of ancient human populations. J Virol 1993;67: 6413-6423.

6 Ong CK, Chan SY, Campo MS, et al: Evolution of human papillomavirus type 18: an ancient phylogenetic root in Africa and intratype diversity reflect coevolution with human ethnic groups. J Virol 1993; 67:6424-6431.

7 Zur Hausen H, de Villiers EM: Human papillomaviruses. Annu Rev Microbiol 1994;48:427-447.

$\checkmark 8$ Burns DA: 'Warts and all' - the history and folklore of warts: a review. J R Soc Med 1992;85:37-40.

9 Rigoni-Stern D: Fatti statistici relative alle mallatie cancrosi che servirono de base alla poche cose dette dal dott. Giornale service propr.pathol.terap.ser. 1842;2:507-517 [Italian].

10 Zur Hausen H: Papillomaviruses in the causation of human cancers - a brief historical account. Virology 2009;384:260-265.

11 Schiffman M, Wentzensen N: Human papillomavirus infection and the multistage carcinogenesis of cervical cancer. Cancer Epidemiol Biomarkers Prev 2013;22:553-560.
12 Schiffman M, Castle PE, Jeronimo J, Rodriguez AC, Wacholder S: Human papillomavirus and cervical cancer. Lancet 2007;370:890-907.

13 Jemal A, Simard EP, Dorell C, et al: Annual report to the nation on the status of cancer, 1975-2009, featuring the burden and trends in human papillomavirus (HPV)-associated cancers and HPV vaccination coverage levels. J Natl Cancer Inst 2013;105:175-201.

14 Jemal A, Bray F, Center MM, Ferlay J, Ward E, Forman D: Global cancer statistics. CA Cancer J Clin 2011;61:69-90.

15 Shope RE, Hurst EW: Infectious papillomatosis of rabbits: with a note on the histopathology. J Exp Med 1933;58:607-624.

16 Rous P, Beard JW: The progression to carcinoma of virus-induced rabbit papillomas (Shope). J Exp Med 1935;62:523-548.

17 zur Hausen H: Human papillomaviruses and their possible role in squamous cell carcinomas. Curr Top Microbiol Immunol 1977;78:1-30.

18 zur Hausen H: Papillomaviruses in anogenital cancer as a model to understand the role of viruses in human cancers. Cancer Res 1989;49:4677-4681.

19 Bernard HU, Burk RD, Chen Z, van Doorslaer K, Hausen H, de Villiers EM: Classification of papillomaviruses (PVs) based on $189 \mathrm{PV}$ types and proposal of taxonomic amendments. Virology 2010;401:70-79.

20 Munoz N, Bosch FX, de Sanjose S, et al: The causal link between human papillomavirus and invasive cervical cancer: a population-based case-control study in Colombia and Spain. Int J Cancer 1992;52:743-749.

-21 Rodriguez AC, Schiffman M, Herrero R, et al: Rapid clearance of human papillomavirus and implications for clinical focus on persistent infections. J Natl Cancer Inst 2008;100:513-517.

22 Denny L, Kuhn L, Hu CC, Tsai WY, Wright TC Jr: Human papillomavirus-based cervical cancer prevention: long-term results of a randomized screening trial. J Natl Cancer Inst 2010;102:1557-1567. 
23 Barzon L, Militello V, Lavezzo E, et al: Human papillomavirus genotyping by 454 next generation sequencing technology. J Clin Virol 2011;52:93-97.

24 Mighty KK, Laimins LA: The role of human papillomaviruses in oncogenesis. Recent Results Cancer Res 2014;193:135-148.

25 Doorbar J, Quint W, Banks L, et al: The biology and life-cycle of human papillomaviruses. Vaccine 2012; 30:F55-F70.

26 Johansson C, Schwartz S: Regulation of human papillomavirus gene expression by splicing and polyadenylation. Nat Rev Microbiol 2013;11:239-251.

27 Chen Z, Schiffman M, Herrero R, et al: Evolution and taxonomic classification of human papillomavirus 16 (HPV16)-related variant genomes: HPV31, HPV33, HPV35, HPV52, HPV58 and HPV67. PloS One 2011;6:e20183.

28 Klug A, Finch JT: Structure of viruses of the papilloma-polyoma type. I. Human wart virus. J Mol Biol 1965;11:403-423.

29 Klug A, Finch JT: Structure of viruses of the papilloma-polyoma type. IV. Analysis of tilting experiments in the electron microscope. J Mol Biol 1968;31:1-12.

30 Gissmann L, Pfister H, Zur Hausen H: Human papilloma viruses (HPV): characterization of four different isolates. Virology 1977;76:569-580.

31 Reinson T, Toots M, Kadaja M, et al: Engagement of the ATR-dependent DNA damage response at the human papillomavirus 18 replication centers during the initial amplification. J Virol 2013;87:951-964.

32 Phelps WC, Howley PM: Transcriptional trans-activation by the human papillomavirus type $16 \mathrm{E} 2$ gene product. J of virol 1987;61:1630-1638.

33 Bedrosian CL, Bastia D: The DNA-binding domain of HPV-16 E2 protein interaction with the viral enhancer: protein-induced DNA bending and role of the nonconserved core sequence in binding site affinity. Virology 1990;174:557-575.

34 Hegde RS: The papillomavirus E2 proteins: structure, function, and biology. Annu Rev Biophys Biomol Struct 2002;31:343-360.

-35 Fu L, Van Doorslaer K, Chen Z, et al: Degradation of p53 by human Alphapapillomavirus E6 proteins shows a stronger correlation with phylogeny than oncogenicity. PloS One 2010;5:e12816.

36 Chen SL, Mounts P: Transforming activity of E5a protein of human papillomavirus type 6 in NIH 3T3 and C127 cells. J Virol 1990;64:3226-3233.

37 Hwang ES, Nottoli T, Dimaio D: The HPV16 E5 protein: expression, detection, and stable complex formation with transmembrane proteins in COS cells. Virology 1995;211:227-233.

38 Schiffman M, Herrero R, Desalle R, et al: The carcinogenicity of human papillomavirus types reflects viral evolution. Virology 2005;337:76-84.
39 Zur Hausen H, Meinhof W, Scheiber W, Bornkamm GW: Attempts to detect virus-specific DNA in human tumors. I. Nucleic acid hybridizations with complementary RNA of human wart virus. Int J Cancer 1974;13:650-656.

40 Boshart M, Gissmann L, Ikenberg H, Kleinheinz A, Scheurlen W, zur Hausen H: A new type of papillomavirus DNA, its presence in genital cancer biopsies and in cell lines derived from cervical cancer. EMBO J 1984;3:1151-1157.

41 Van Ranst M, Fuse A, Sobis H, et al: A papillomavirus related to HPV type 13 in oral focal epithelial hyperplasia in the pygmy chimpanzee. J Oral Pathol Med 1991;20:325-331.

42 Chan SY, Bernard HU, Ratterree M, Birkebak TA, Faras AJ, Ostrow RS: Genomic diversity and evolution of papillomaviruses in rhesus monkeys. J Virol 1997;71:4938-4943.

43 Chen Z, van Doorslaer K, DeSalle R, et al: Genomic diversity and interspecies host infection of alpha12 Macaca fascicularis papillomaviruses (MfPVs). Virology 2009;393:304-310.

44 Bergin IL, Bell JD, Chen Z, et al: Novel genital Alphapapillomaviruses in baboons (Papio hamadryas anubis) with cervical dysplasia. Vet Pathol 2013;50: 200-208.

45 Wood CE, Chen Z, Cline JM, Miller BE, Burk RD: Characterization and experimental transmission of an oncogenic papillomavirus in female macaques. J Virol 2007;81:6339-6345.

46 Chen Z, DeSalle R, Schiffman M, Herrero R, Burk $\mathrm{RD}$ : Evolutionary dynamics of variant genomes of human papillomavirus types 18,45 , and 97 . J Virol 2009;83:1443-1455.

47 Rector A, Lemey P, Tachezy R, et al: Ancient papillomavirus-host co-speciation in Felidae. Genome Biol 2007;8:R57.

48 Narechania A, Chen Z, DeSalle R, Burk RD: Phylogenetic incongruence among oncogenic genital alpha human papillomaviruses. J Virol 2005;79:15503-15510.

49 Burk RD, Chen Z, Van Doorslaer K: Human papillomaviruses: genetic basis of carcinogenicity. Public Health Genom 2009;12:281-290.

50 Schiffman M, Rodriguez AC, Chen Z, et al: A population-based prospective study of carcinogenic human papillomavirus variant lineages, viral persistence, and cervical neoplasia. Cancer Res 2010;70: 3159-3169.

51 De Villiers EM: Heterogeneity of the human papillomavirus group. J Virol 1989;63:4898-4903.

52 Qu W, Jiang G, Cruz Y, et al: PCR detection of human papillomavirus: comparison between MY09/ MY11 and GP5+/GP6+ primer systems. J Clin Microbiol 1997;35:1304-1310.

53 Abreu AL, Souza RP, Gimenes F, Consolaro ME: A review of methods for detect human papillomavirus infection. Virol J 2012;9:262. 
54 De Villiers EM: Cross-roads in the classification of papillomaviruses. Virology 2013;445:2-10.

55 Burk RD, Harari A, Chen Z: Human papillomavirus genome variants. Virology 2013;445:232-243.

56 Bernard HU: The clinical importance of the nomenclature, evolution and taxonomy of human papillomaviruses. J Clin Virol 2005;32(suppl 1):S1-S6.

57 Chen Z, Terai M, Fu L, Herrero R, DeSalle R, Burk RD: Diversifying selection in human papillomavirus type 16 lineages based on complete genome analyses. J Virol 2005;79:7014-7023.

58 Gottschling M, Kohler A, Stockfleth E, Nindl I: Phylogenetic analysis of beta-papillomaviruses as inferred from nucleotide and amino acid sequence data. Mol Phylogenet Evol 2007;42:213-222.

-59 Egawa K, Delius H, Matsukura T, Kawashima M, de Villiers EM: Two novel types of human papillomavirus, HPV 63 and HPV 65: comparisons of their clinical and histological features and DNA sequences to other HPV types. Virology 1993;194:789-799.

60 Bottalico D, Chen Z, Dunne A, et al: The oral cavity contains abundant known and novel human papillomaviruses from the Betapapillomavirus and Gammapapillomavirus genera. J Infect Dis 2011;204:787-792.

61 Gage JC, Schiffman M, Solomon D, et al: Risk of precancer determined by HPV genotype combinations in women with minor cytologic abnormalities. Cancer Epidemiol Biomarkers Prev 2013;22:1095-1101.

62 Burk RD, Terai M, Gravitt PE, et al: Distribution of human papillomavirus types 16 and 18 variants in squamous cell carcinomas and adenocarcinomas of the cervix. Cancer Res 2003;63:7215-7220.

63 Li N, Franceschi S, Howell-Jones R, Snijders PJ, Clifford GM: Human papillomavirus type distribution in 30,848 invasive cervical cancers worldwide: Variation by geographical region, histological type and year of publication. Int J Cancer 2011;128:927-935.

$\checkmark 64$ Chen Z, Schiffman M, Herrero R, et al: Evolution and Taxonomic Classification of Alphapapillomavirus 7 Complete Genomes: HPV18, HPV39, HPV45, HPV59,HPV68 and HPV70. PloSOne 2013;8:e72565.

65 Chan SY, Ho L, Ong CK, et al: Molecular variants of human papillomavirus type 16 from four continents suggest ancient pandemic spread of the virus and its coevolution with humankind. J Virol 1992;66:20572066.

66 Smith B, Chen Z, Reimers L, et al: Sequence imputation of HPV 16 genomes for genetic association studies. PloS One 2011;6:e21375.

Robert D. Burk

Albert Einstein College of Medicine

1300 Morris Park Avenue

Bronx, NY 10461 (USA)

E-Mail robert.burk@einstein.yu.edu
67 Chan SY, Chew SH, Egawa K, et al: Phylogenetic analysis of the human papillomavirus type 2 (HPV2), HPV-27, and HPV-57 group, which is associated with common warts. Virology 1997;239:296-302.

68 Yamada T, Manos MM, Peto J, et al: Human papillomavirus type 16 sequence variation in cervical cancers: a worldwide perspective. J Virol 1997;71:24632472.

69 Guan P, Howell-Jones R, Li N, et al: Human papillomavirus types in 115,789 HPV-positive women: a meta-analysis from cervical infection to cancer. Int J Cancer 2012;131:2349-2359.

70 Castle PE, Porras C, Quint WG, et al: Comparison of two PCR-based human papillomavirus genotyping methods. J Clin Microbiol 2008;46:3437-3445.

71 Xi LF, Schiffman M, Koutsky LA, et al: Persistence of newly detected human papillomavirus type 31 infection, stratified by variant lineage. Int J Cancer 2013; 132:549-555.

72 Rositch AF, Koshiol J, Hudgens MG, et al: Patterns of persistent genital human papillomavirus infection among women worldwide: a literature review and meta-analysis. Int J Cancer 2013;133:1271-1285.

73 Xi LF, Schiffman M, Koutsky LA, et al: Association of human papillomavirus type 31 variants with risk of cervical intraepithelial neoplasia grades 2-3. Int J Cancer 2012;131:2300-2307.

74 Conway C, Chalkley R, High A, et al: Next-generation sequencing for simultaneous determination of human papillomavirus load, subtype, and associated genomic copy number changes in tumors. J Mol Diagn 2012;14:104-111.

75 Foulongne V, Sauvage V, Hebert C, et al: Human skin microbiota: high diversity of DNA viruses identified on the human skin by high throughput sequencing. PloS One 2012;7:e38499.

76 Johansson H, Bzhalava D, Ekstrom J, Hultin E, Dillner J, Forslund O: Metagenomic sequencing of 'HPV-negative' condylomas detects novel putative HPV types. Virology 2013;440:1-7.

77 Clarke MA, Wentzensen N, Mirabello L, et al: Human papillomavirus DNA methylation as a potential biomarker for cervical cancer. Cancer Epidemiol Biomarkers Prev 2012;21:2125-2137.

78 Mirabello L, Schiffman M, Ghosh A, et al: Elevated methylation of HPV16 DNA is associated with the development of high grade cervical intraepithelial neoplasia. Int J Cancer 2013;132:1412-1422.

79 Mirabello L, Sun C, Ghosh A, et al: Methylation of human papillomavirus type 16 genome and risk of cervical precancer in a Costa Rican population. J Natl Cancer Inst 2012;104:556-565.

80 Stamatakis A: RAxML-VI-HPC: maximum likelihood-based phylogenetic analyses with thousands of taxa and mixed models. Bioinformatics 2006;22: 2688-2690. 